\title{
Caracterización de los Sistemas Ganaderos de Aptitud Lechera en el Valle del Quijos, Provincia del Napo, Ecuador
}

\author{
Santiago Alexander Guamán Rivera, M.Sc \\ Estudiante de Doctorado en Producción Animal Universidad Autónoma de \\ Barcelona (España) - Grupo de Investigación en Rumiantes (G2R) \\ Departamento de Ciencia Animal y Alimentos, Escuela Superior Politécnica \\ de Chimborazo, Extensión Norte Amazónica, Ecuador \\ Raúl Lorenzo González Marcillo, M.Sc \\ Robinson Carrasco, M.Sc \\ Fabián Guamán, M.Sc. \\ Escuela Superior Politécnica de Chimborazo, \\ Extensión Norte Amazónica, Ecuador
}

Doi: 10.19044/esj.2019.v15n15p279 URL:http://dx.doi.org/10.19044/esj.2019.v15n15p279

\section{Resumen}

Este trabajo permitió identificar y caracterizar la estructura, función, así como el uso de tecnología de los sistemas ganaderos, sobre las dinámicas espaciales, socioeconómicas y ambientales en las cuales se desarrolla la ganadería en el Valle del Quijos (Ecuador). La metodología se basó en la exploración bibliográfica y el trabajo de campo por medio de observación y encuestas aplicadas en 70 fincas de la región. Como resultado del análisis estadístico, se caracterizó la existencia de cuatro tipologías ganaderas; G1 sin uso de tecnología, G2 poco uso de tecnología, G3 semitecnificadas, G4 tecnificadas. En las tres primeras ( $93.17 \%$ del total), el componente boscoso cubre un porcentaje muy alto en relación con el tamaño de las fincas, encontrando en estos grupos sistemas silvopastoriles (SSP) en altas densidades de árboles por hectárea bajo arreglos espaciales como cercas vivas y dispersos en potreros. Igualmente tenemos varios biotipos bovinos, en donde para las primeras tipologías ganaderas G1, G2 y G3, predominan animales mestizos de razas (Brown Swiss, Holstein, Normando) con orientación productiva doble propósito (carne y leche); la composición del hato muestra una distribución atípica en las dos primeras tipologías, al no existir equilibrio de acuerdo con cada categoría bovina, afectando así la productividad. Mientras tanto en el grupo de alto uso de tecnología, se tienen bovinos de razas de producción de leche puras o en cruces (Jersey, Holstein). Se concluye que existe una amplia 
divergencia entre los sistemas ganaderos identificados y caracterizados en el Valle del Quijos y que, a medida que se ha ganado en intensificación, se ha perdido en conservación.

Palabras Claves: Ganadería lechera, sistemas silvopastoriles, producción animal, estadística, medioambiente

\title{
Characterization of Livestock Aptitude Cattle Systems in the Quijos Valley, Napo Province, Ecuador
}

\author{
Santiago Alexander Guamán Rivera, M.Sc \\ Estudiante de Doctorado en Producción Animal Universidad Autónoma de \\ Barcelona (España) - Grupo de Investigación en Rumiantes (G2R) \\ Departamento de Ciencia Animal y Alimentos, Escuela Superior Politécnica \\ de Chimborazo, Extensión Norte Amazónica, Ecuador \\ Raúl Lorenzo González Marcillo, M.Sc \\ Robinson Carrasco, M.Sc \\ Fabián Guamán, M.Sc. \\ Escuela Superior Politécnica de Chimborazo, \\ Extensión Norte Amazónica, Ecuador
}

\begin{abstract}
This paper focuses on identifying and characterizing the structure, function, as well as the use of technology of the livestock systems on the spatial, socioeconomic, and environmental dynamics in which livestock is developed in the Quijos Valley (Ecuador). The methodology was based on bibliographic exploration and fieldwork through observation and surveys applied in 70 farms in the region. As a result of the statistical analysis, the existence of four livestock typologies was characterized; G1 without the use of technology, G2 little use of technology, G3 semi-technified, G4 technified. In the first three ( $93.17 \%$ of the total), the forest component covers a very high percentage in relation to the size of the farms. In these groups, it shows silvopastoral systems (SSP) in high densities of trees per hectare under spatial arrangements such as live fences and scattered paddocks. We also have several bovine biotypes. For the first livestock typologies G1, G2 and G3, mestizo breed animals (Brown Swiss, Holstein, Norman) predominate with dual
\end{abstract}


purpose productive orientation (meat and milk). The composition of the herd shows an atypical distribution in the first two typologies. This is because there is no balance according to each bovine category, thus having a significant effect on productivity. Meanwhile in the group of high use of technology, there are bovine animals of pure milk production or crosses (Jersey, Holstein). It is concluded that there is a wide divergence between the livestock systems identified and characterized in the Quijos Valley. Thus, the measure gained in intensification has been lost in conservation.

Keywords: Dairy farming, silvopastoral systems, animal production, statistics, environment

\section{Introduction}

Diferentes estudios sugieren que la rápida expansión de la ganadería y el uso extensivo de prácticas no sostenibles de manejo de pasturas son probablemente los mayores determinantes de la deforestación y la degradación ambiental en áreas rurales de la Amazonía continental (Grijalva, Arévalo, \& Barrera, 2002; Wunder, 2000; Veiga \& Tourrand, 2001; Ferreira \& Tourrand, 2002; Grijalva, Arévalo, \& Wood, 2004; Ríos-Núñez \& BenítezJiménez, 2015). Estas formas inadecuadas de uso del suelo han conducido a la degradación de los recursos naturales y a la pérdida de las cuencas hidrográficas y de la biodiversidad, con impactos drásticos en las oportunidades de subsistencia de las poblaciones más vulnerables de la región (Pérez Sánchez, 2006; Pérez Espejo, 2008; Vargas, Ríos, Torres, Benítez, Soria, Navarrete, \& Pardo, 2014; Anda, Gómez de la Torre \& Bedoya, 2017).

La cobertura boscosa original de la Amazonía estaba alrededor de 6,1 millones de $\mathrm{km} 2: 41,2 \%$ en la Amazonía andina y guayanesa y 58,8 \% en Brasil. Hasta el año 2000, se había perdido alrededor de 9,7\% de ese total, donde Brasil lideraba las mayores pérdidas con un 12,8\%, seguido por Ecuador con $9,6 \%$ y luego Colombia y Perú con 7,4 y 7,0\%, respectivamente (Oliveira-Miranda, 2015).

Las cifras a nivel de país señalan un ritmo de deforestación que fluctúa entre 130000 y 230000 hectáreas, cifra que equivale al 1,2 - 1,8\% de la superficie con bosques. Más del $70 \%$ de la madera aprovechada y comercializada es ilegal y proviene del bosque húmedo tropical de la Amazonía (Pérez Espejo, 2008; Albán, 2010; Mogrovejo Jaramillo, 2017).

El proyecto IAI/NSF Ganadería, uso de la tierra y deforestación en la Amazonía: estudio comparativo entre Brasil, Perú y Ecuador, desarrollado entre 2000 y 2002 (Grijalva et al., 2002), constituyó una iniciativa regional en la que participaron la Universidad de Florida (EE. UU.), la Empresa Brasileña de Pesquisas Agropecuarias (EMBRAPA), el Instituto Nacional Autónomo de Investigaciones Agropecuarias (INIAP), la Universidad 
Nacional Agraria de la Selva (UNAS) y la Universidad Federal de Pará. Esa iniciativa se ejecutó con el auspicio del Inter American Institute for Global Change Research (IAI) y el cofinanciamiento de la National Science Foundation (NSF). Su propósito fue analizar comparativamente la expansión del ganado y sus consecuencias sobre la deforestación en la cuenca amazónica de Brasil, Perú y Ecuador. En ese marco, el estudio en el Ecuador se focalizó en el análisis de los factores socioeconómicos y biofísicos que influyen en el proceso de toma de decisiones por parte de una diversidad de actores rurales, quienes deciden invertir su tierra, mano de obra y capital en la formación de pasturas y la ganadería en dos contextos contrastantes de esta región: el Valle del Quijos y las zonas de piedemonte en Napo, y la región del Coca en Francisco de Orellana (Grijalva et al., 2004; Ochoa \& Valarezo-García, 2014).

El resultado más relevante de ese estudio determinó que la vinculación del Valle de Quijos al mercado, mediante el proceso de colonización, desencadenó una dinámica de diferenciación campesina y la configuración de una estructura productiva diversa, ligada principalmente a la actividad lechera. Esta diferenciación se refleja en una dualidad caracterizada por la convergencia de economías campesinas y de economías de tipo mercantil. Las primeras, marginales y de subsistencia, están poco articuladas al mercado y en ellas persiste una lógica de valorización precapitalista que depende de la fuerza laboral familiar con un bajo nivel tecnológico. Las segundas, estabilizadas y empresariales, articuladas estrechamente al mercado, utilizan mano de obra asalariada y presentan distintos niveles tecnológicos y acumulación de capital (Grijalva et al., 2004; Arévalo, Andino \& Grijalva, 2008; Ochoa \& Valarezo-García, 2014).

A partir de estos antecedentes, se realizó una investigación orientada a analizar las dinámicas espaciales, socioeconómicas y ambientales en las cuales se desarrolla la ganadería a lo largo del Valle de Quijos, dada la relevancia socioeconómica de esta actividad para la población del sector y su entorno. El presente artículo identifica, caracteriza y describe entonces la estructura y función, así como el uso de tecnología de los sistemas ganaderos en el Valle del Quijos, Ecuador.

\section{Metodología}

\section{Características de la zona de estudio}

El Valle del Quijos se encuentra ubicado al nororiente de Ecuador. Pertenece territorialmente a la Provincia del Napo, ubicado en las estribaciones de la cordillera de los Andes y en la selva alta de la región amazónica. Forma parte de la cuenca hidrográfica alta del río Napo y de la microcuenca de los ríos Papallacta, Quijos y Cosanga. Ubicado sobre la Cordillera Oriental de los Andes, se encuentra rodeado de páramos, matorrales 
y bosques e innumerables lagunas que forman parte de las áreas protegidas de la Reserva Ecológica Antisana, la Reserva Ecológica Cayambe-Coca y el Parque Nacional Sumaco-Napo Galeras. Con referencia a su temperatura, el Valle de Quijos depende del piso altitudinal; el promedio se encuentra entre $9,4^{\circ} \mathrm{C}$ y $16,2^{\circ} \mathrm{C}$. Su humedad relativa está entre $85 \%$ y $93 \%$, la heliofanía es de 2646 mm y su evaporación es de 587,2 (Cañadas, 1983).

El Valle del Quijos territorialmente está constituido por el cantón Quijos, conformado por las parroquias Baeza, Cosanga, Cuyuja, Papallacta, San Francisco de Borja y Sumaco, y por el cantón El Chaco, parroquias El Chaco, Gonzalo Díaz de Pineda, Linares, Santa Rosa y Sardinas.

\section{Selección y tamaño de la muestra}

La población objetivo se estableció con base en el listado de productores ganaderos registrados por el Consejo Nacional de Erradicación de la Fiebre Aftosa (CONEFA) y el Ministerio de Agricultura, Ganadería, Acuacultura y Pesca (MAGAP) de Ecuador durante las campañas de vacunación y reactivación de pastos. Para la determinación del tamaño de la muestra se utilizó la metodología de Aguilar-Barojas (2005) y se aplicó esta ecuación:

$$
n=\frac{N Z^{2} \sigma^{2}}{e^{2}(N-1)+Z^{2} \sigma^{2}}
$$

El significado de las variables es el siguiente:

$\mathrm{N}=$ Tamaño de la población

$\mathrm{Z}=$ Valor obtenido de la tabla Z (Grijalva et al., 2004, p. 34)

$\mathrm{e}=$ Error permisible

$\sigma 2=$ Varianza de la población

Los valores de las variables se reemplazan así:

$\mathrm{N}=1066$ unidades productivas agropecuarias (UPA)

$\mathrm{Z}=1,96$

$\mathrm{e}=1 / 3$ de litros/vaca/día

$\sigma 2=(1.5)^{2}$

El error permisible para tener un rango de confiabilidad es de un máximo de $1 / 3$ de litros/vaca/día, el cual se obtuvo mediante el estudio de Grijalva et al. (2004). La variable producción de leche/vaca/día tiene una distribución normal. E1 $95 \%$ de la población está comprendida en un rango de producción equivalente a 5 litros/vaca/día y un máximo de 11 litros/vaca/día; 
el promedio es 8 litros/vaca/día. Como se trabajó con el $95 \%$ de probabilidad, hay una significación del 0,05 y Grijalva et al. (2004) propone que el valor correspondiente de $\mathrm{Z}$ para esa significación es 1,96 (Grijalva et al., 2004, p. 34). El resultado de la ecuación fue aproximado a 70 fincas.

La matriz de la encuesta se construyó con variables de tipo estructural, funcional y socioeconómico: identificación, usos del suelo, medios de producción, servicios básicos, superficie dedicada a pastos y forrajes, intensidad de pastoreo, determinación de carga animal, determinación de la capacidad de carga, componente animal, agrícola y forestal, situación socioeconómica, ingresos extrafinca y acceso a crédito.

Las encuestas se validaron en campo en varias UPA en conjunto con un equipo multidisciplinar para determinar la pertinencia de las preguntas. A cada respuesta cualitativa se le asignó un código numérico y a las variables cuantitativas se les asignó sus unidades numéricas correspondientes. Luego de validadas, se aplicaron las encuestas a 70 fincas de manera aleatoria en diferentes parroquias del Valle del Quijos (Tabla 1).

Tabla 1. Encuestas aplicadas en las diferentes parroquias de los cantones Quijos y el Chaco, pertenecientes al Valle del Quijos, Napo, Ecuador

\begin{tabular}{|c|c|c|c|}
\hline Cantón & Parroquia & Cantidad & $\mathbf{( ~ \% )}$ \\
\hline \multirow{4}{*}{ Quijos } & Papallacta & 2 & 2,85 \\
\cline { 2 - 4 } & Cuyuja & 7 & 10 \\
\cline { 2 - 4 } & Baeza & 14 & 20 \\
\cline { 2 - 4 } & Borja & 9 & 12,85 \\
\cline { 2 - 4 } & Sumaco & 3 & 4,28 \\
\cline { 2 - 4 } & Cosanga & 11 & 15,71 \\
\cline { 2 - 4 } & Sardinas & 7 & 10 \\
\hline \multirow{4}{*}{ Chaco } & Chaco & 2 & 2,85 \\
\cline { 2 - 4 } & Linares & 4 & 5,71 \\
\cline { 2 - 4 } & Santa Rosa & 8 & 11,42 \\
\cline { 2 - 4 } & Gonzalo Díaz & 3 & 4,28 \\
\hline \multicolumn{1}{|c|}{} & $\mathbf{7 0}$ & $\mathbf{1 0 0}$ \\
\hline
\end{tabular}

\section{Análisis estadístico}

Los datos fueron procesados mediante el programa estadístico SPSS, versión 17 para Windows. Se utilizó la técnica multivariante de componentes principales y el cálculo de estadística descriptiva (promedios, desviaciones estándar, coeficientes de variación, mínimos y máximos). Se tipificaron los sistemas ganaderos mediante el análisis de conglomerados.

Una vez obtenidos los valores correspondientes a la varianza de cada variable y luego de haber encontrado la comunalidad, que es extraída por el programa estadístico, se seleccionaron las variables con un CV (coeficiente de variabilidad) $\pm>30$ para construir el dendograma (ver Tabla 2). El resto se 
descartaron. De esta manera se eligió información homogénea y de baja comunalidad.

Tabla 2. Variables seleccionadas de acuerdo con su CV para realizar la tipificación de los productores del Valle del Quijos

\begin{tabular}{|l|c|c|}
\hline \multicolumn{1}{|c|}{ Variables } & SD $^{\mathbf{1}}$ & $\mathbf{C V}^{\mathbf{2}}$ \\
\hline v. 1: Propietario & 20,35 & 57,47 \\
\hline v. 2: Tamaño de la finca & 28,63 & 85,16 \\
\hline v. 3: Vacas en producción & 7,15 & 67,81 \\
\hline v. 4: Total litros/día & 75,98 & 95,29 \\
\hline v. 5: Mano de obra familiar & 0,79 & 47,83 \\
\hline v. 6: Mano de obra permanente & 1,29 & 62,79 \\
\hline v. 7: Mano de obra ocasional & 86,60 & 144,79 \\
\hline v. 8: Suplementación animal & 0,28 & 28,20 \\
\hline v. 9: Superficie con cultivos & 0,91 & 72,84 \\
\hline v. 10: Árboles dispersos en potreros & 0,34 & 33,71 \\
\hline v. 11: SSP de árboles dispersos en potreros & 0,34 & 33,71 \\
\hline v. 12: SSP de cercas vivas & 0,51 & 50,71 \\
\hline v. 13: SSP de bosquetes & 0,50 & 50,18 \\
\hline v. 14: SSP de linderos & 0,23 & 29,22 \\
\hline v. 15: Ingresos extrafinca & 0,49 & 49,03 \\
\hline v. 16: Ingresos de la finca & 0,50 & 49,84 \\
\hline v. 17: Acceso a crédito & 0,34 & 33,71 \\
\hline v. 18: Toma de decisiones pecuarias & 1,00 & 49,99 \\
\hline v. 19: Toma de decisiones socioeconómicas & 0,79 & 30,35 \\
\hline v. 20: Toma de decisiones forestales & 0,99 & 52,64 \\
\hline
\end{tabular}

${ }^{1}$ Desviación estándar (Standard deviation).

${ }^{2}$ Coeficiente de variación.

\section{Resultados y discusión}

Analizados los datos, identificamos la existencia de cuatro grupos de ganaderías en el Valle del Quijos: ganaderías sin uso de tecnología (G1), ganaderías poco tecnificadas $(\mathrm{G} 2)$, ganaderías semitecnificadas (G3) y ganaderías tecnificadas (G4). En la Tabla 3 se observan el porcentaje de representación de cada una de estas tipologías. La de mayor representación es la $\mathrm{G} 1$ con un $82,85 \%$.

Tabla 3. Tipificación de las ganaderías del Valle del Quijos de acuerdo con grado de tecnificación

\begin{tabular}{|c|c|c|c|}
\hline Grupo & Ganaderías & N. ${ }^{\circ}$ fincas & $\%$ \\
\hline G1 & Ganaderías sin uso de tecnología & 58 & 82,85 \\
\hline $\mathrm{G} 2$ & Ganaderías poco tecnificadas & 6 & 8,57 \\
\hline G3 & Ganaderías semitecnificadas & 4 & 5,71 \\
\hline G4 & Ganaderías tecnificadas & 2 & 2,85 \\
\hline \multicolumn{2}{|r|}{ Total } & 70 & 100 \\
\hline
\end{tabular}

G1: Las ganaderías de esta tipología están ubicadas en zonas próximas a reservas naturales. El $83 \%$ de este grupo tiene sus fincas legalizadas, el $11 \%$ no cuenta con títulos de propiedad y el $6 \%$ restante está bajo contratos de 
arrendamiento. Esta información se contrastó con la de Leiva (2011) y se halló que los resultados fueron similares.

En este grupo G1 se destacan los sistemas agroforestales, especialmente los silvopastoriles (árboles en cercas vivas, bosques secundarios, arboles dispersos en potreros y árboles en linderos). Dichos sistemas tienen una alta relevancia para este tipo de ganaderías (Ochoa \& Valarezo-García, 2014), debido principalmente a los beneficios ambientales.

Este tipo de fincas mantienen y crían bovinos mestizos para doble propósito (leche y carne). Para su alimentación introducen pasturas forrajeras que carecen de alto valor nutricional (Vargas et al., 2011).

Se ha etiquetado este grupo como de bajo nivel tecnológico debido a que en sus actividades cotidianas no usan equipos ni instrumentos técnicos (Carrasco et al., 2017).

G2: En relación con el uso del suelo, las fincas ganaderas de este grupo poseen bosques secundarios por regeneración natural. En estas tipologías, el $100 \%$ de las propiedades están debidamente legalizadas. Se encuentran en menor porcentaje que respecto al G1 especies forestales en arreglos agroforestales de árboles dispersos en potreros y en cercas vivas. Manejan un hato con cruces de razas Holstein y Brown Swiss con genotipos criollos o evolucionados en el trópico o la Amazonía que se adaptan mejor a las condiciones adversas del entorno para la producción de leche (Vargas et al., 2014). Para las actividades de la finca usan herramientas manuales y una que otra herramienta mecánica como la guadaña y la motosierra.

G3: En comparación con los dos grupos anteriores, la extensión ocupada por bosques secundarios en los sistemas del G3 se reduce considerablemente para la ampliación de especies forrajeras destinadas para la alimentación animal. Con respecto a la tenencia de la tierra, el $75 \%$ de las fincas tienen legalizadas sus propiedades. Manejan hatos bovinos con biotipos de aptitud lechera. Las ganaderías de este grupo, además de las herramientas manuales, ya cuentan con cerca eléctrica, guadaña y motosierra.

G4: Las fincas de este grupo tienen una superficie promedio de 42 hectáreas ocupadas por especies forrajeras destinadas a la alimentación de bovinos de razas de aptitud lechera (debidamente registrados).Encontramos árboles en sistemas silvopastoriles bajo arreglos espaciales como lo son árboles dispersos en potreros pero en bajas densidades (1,8 árboles/ha) en comparación con las ganaderías anteriores (G1: 136 árboles/ha, G2: 80 árboles/ha y G3 16 árboles/ha). Las ganaderías del grupo G4 han adaptado y utilizan equipos y herramientas de sistemas intensivos de la región Sierra Centro del Ecuador. Se emplea ordeñadora mecánica, tanques de enfriamiento de leche, inseminación artificial y cercas eléctricas, además de herramientas manuales y mecánicas como la guadaña y la motosierra. Carrasco et al. (2017) plantearon que la necesidad de superficie especializada se contrapone a los 
criterios de conservación, donde prima la tecnificación. El ambiente es dejado en segundo plano y el componente boscoso desaparece. Económicamente es más rentable convertir superficies de bosque en potreros.

\section{Superficie, uso del suelo y tenencia de la tierra}

Respecto al tamaño de las fincas (Tabla 4), determinamos que, a mayor extensión de tierra, más hectáreas destinadas al uso con pasturas. En las fincas poco tecnificadas, dadas sus condiciones, se aumenta la superficie para uso de la ganadería en detrimento de la parte boscosa (ESPAC, 2017). En el grupo G1, pese a tener una extensión promedio de 27 hectáreas, el $73 \%$ está cubierto por pastos. El mismo proceso siguen las de tipo G2, G3 y G4, en donde el componente boscoso paulatinamente se desplaza de las fincas para ser destinado al uso de pasturas (Ríos-Núñez \& Benítez-Jiménez, 2015). Carrasco et al. (2017) demostraron que la tenencia de la tierra en Ecuador es un factor condicionante de la presencia de sistemas ganaderos de mayor o menor grado de intensificación y determinantes de las necesidades de formación de los ganaderos en procesos de extensión rural.

Tabla 4. Superficie, uso del suelo y tenencia de la tierra de las tipologías de ganaderías identificadas y caracterizadas en el Valle del Quijos

\begin{tabular}{|l|c|c|c|c|c|}
\hline \multirow{2}{*}{$\begin{array}{c}\text { Superficie, uso del suelo y } \\
\text { tenencia de la tierra }\end{array}$} & \multicolumn{4}{|c|}{ Tipologías } \\
\cline { 2 - 6 } & G1 & G2 & G3 & G4 \\
\hline \pm de hectáreas* & $\mathrm{Ha}$ & $27 \mathrm{ha}$ & $89,8 \mathrm{ha}$ & $39 \mathrm{ha}$ & $42,5 \mathrm{ha}$ \\
\hline Bosques & $\%$ & $26 \%$ & $25 \%$ & $21 \%$ & - \\
\hline Pasturas & $\%$ & $73 \%$ & $74 \%$ & $74 \%$ & $100 \%$ \\
\hline Cultivos & $\%$ & $1 \%$ & $1 \%$ & $5 \%$ & - \\
\hline Propia con título & $\%$ & $83 \%$ & $100 \%$ & $75 \%$ & $100 \%$ \\
\hline Propia sin título & $\%$ & $11 \%$ & - & $25 \%$ & - \\
\hline
\end{tabular}

*Dato obtenido por el programa SPSS

\section{Integración de especies forestales con pasturas}

Identificamos en las fincas sistemas silvopastoriles (SSP). Entre ellos destacan arreglos espaciales de manera secuencial o temporal (Lyngbaek, Muschler, \& Sinclair, 2001; Villavicencio-Enríquez, 2013) como árboles dispersos en potreros, cercas vivas, remanentes boscosos y árboles en linderos. Las densidades de estas ganaderías están ligadas a la tipificación de cada grupo de ganaderías: mientras más intensivas, menos densidad de árboles por hectárea. Los sistemas agroforestales diversifican la producción con el fin de generar bienes y servicios que satisfagan las necesidades básicas de los productores y prevean beneficios socioeconómicos y ecológicos (Ramachandran, 1997). La ganadería en el trópico, especialmente en América Latina, se ha caracterizado por una baja eficiencia productiva (Alonso, 2011; Mahecha, 2003). Sin embargo, los SSP han demostrado ser importantes para 
mejorar las condiciones productivas en muchas de las áreas ganaderas (Tabla 5).

Tabla 5. Integración de especies forestales en diferentes arreglos espaciales silvopastoriles encontradas en las tipologías de ganaderías del Valle del Quijos

\begin{tabular}{|l|c|c|c|c|c|}
\hline \multicolumn{7}{|c|}{ Densidad (árboles/hectárea) } \\
\hline \multicolumn{1}{|c|}{ SSP } & G1 & G2 & G3 & G4 & $\% *$ \\
\hline Árboles dispersos & 136 & 80 & 16 & 1.8 & $14,28 \%$ \\
\hline Cercas vivas & 200 & 133 & - & - & $34,75 \%$ \\
\hline Bosquetes & 350 & 280 & 190 & - & $50,28 \%$ \\
\hline Linderos & 10 & - & - & - & $0,61 \%$ \\
\hline Pastoril & 1 & - & - & - & $0,06 \%$ \\
\hline \multicolumn{1}{|c|}{ Total } & $\mathbf{6 9 7}$ & $\mathbf{4 9 3}$ & $\mathbf{2 0 6}$ & $\mathbf{1 . 8}$ & $\mathbf{1 0 0 \%}$ \\
\hline
\end{tabular}

* Porcentaje sobre el total de hectáreas de las cuatro tipologías

En este estudio hallamos una amplia diversidad de especies leñosas, tanto de rápida propagación como de lento crecimiento. Las variedades leñosas mejoran las condiciones físicoquímicas de los suelos además de contribuir a la generación de servicios ambientales. Por esto resulta necesario implementarlas, mantenerlas y conservarlas como prácticas alternativas de ganadería climáticamente inteligente.

\section{Composición del hato}

Las tipologías de ganaderías identificadas y caracterizadas difieren considerablemente en cuanto a la composición del hato (Figura 1; Ríos-Núñez \& Benítez-Jiménez, 2015). En el G1 no se maneja el hato de forma correcta y proporcional; es decir, de modo que asegure un crecimiento sostenible. La productividad se afecta directamente por el desequilibrio en sus categorías de reposición. En las tipologías de ganaderías con medio y alto uso de tecnología (G3 y G4) se evidenció una mejor distribución de sus producciones, partos, remplazos y descartes de animales. Sin embargo, también se observó que, pese a que existió un ligero aumento del número de animales, las producciones no tuvieron mayores incrementos. Resultado similar a lo reportado por el ESPAC, (2017), que, para la zona oriente, establece una media de producción de 4,29 litros/vaca/día. 


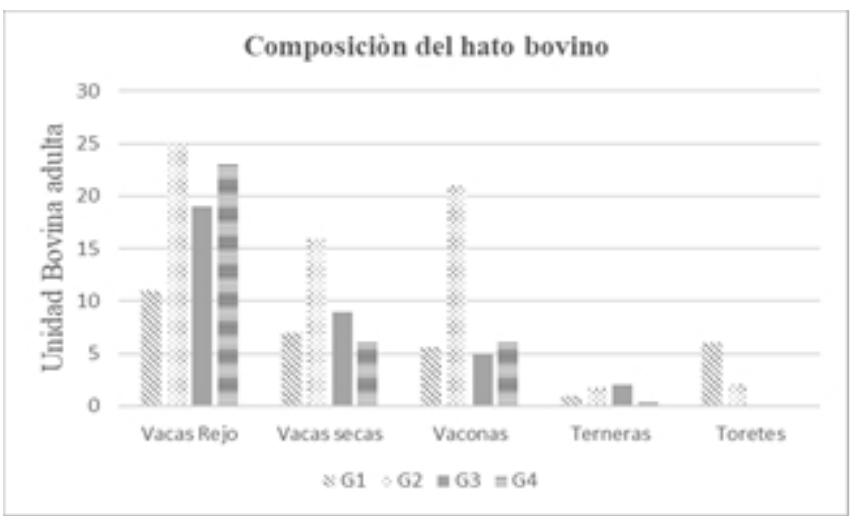

Figura 1. Composición del hato bovino en las diferentes tipologías de ganaderías identificadas y caracterizadas del Valle del Quijos

En síntesism los resultados muestran que en las tres primeras tipologías (97,13\% del total), además del nulo o poco uso de tecnología, hay dos factores comunes. El primero es el componente boscoso, que cubre un porcentaje muy alto en relación con el tamaño de las fincas. El segundo, en relación con el primero, es el uso de sistemas silvopastoriles (SSP) con alta densidad de árboles por hectárea bajo arreglos espaciales dispersos en potreros. En la tipología G4 (sólo dos fincas) hay un alto grado de tecnificación y un acceso amplio a los servicios básicos (luz eléctrica, agua potable, centros educativos y de salud), a los que en general las demás fincas tienen muy poco acceso. Se destaca del estudio que, independientemente del grado de intensificación y tecnificación, en todas las tipologías el componente forrajero está compuesto por gramíneas introducidas y adaptadas al medio cuyo volumen de biomasa varía de acuerdo con el aporte de fuentes inorgánicas así como de labores culturales realizadas. Otro aspecto que resalta del estudio es que la composición del hato muestra una distribución atípica en las fincas tipo G1 y G2: no hay un equilibrio de acuerdo con cada categoría bovina (vacas rejo, vacas secas, vaconas, terneras, toretes), lo que implica que no hay un crecimiento sostenible del hato y que la productividad es muy poca por no haber una reposición. Se evidenció de igual manera la introducción de biotipos bovinos de razas de climas templados para incrementar los rendimientos lecheros.

\section{Conclusiones}

Luego de identificadas y caracterizadas las fincas ganaderas del Valle del Quijos, es posible concluir que existe una amplia divergencia entre ellas y que, a medida que se ha ganado en intensificación, se ha perdido en conservación, lo que conlleva a un problema a mediano y largo plazo por detrimento de la pérdida de bosques. Las tipologías G1, G2 y G3 en su estructura productiva cuentan con el componente boscoso bajo arreglos 
espaciales de SSP en proporciones considerables en relación con el tamaño total de las fincas. Esta información resulta relevante en estos momentos en que se busca la conservación ambiental, principalmente en áreas ecológicas como la del Valle del Quijos.

\section{Agradecimientos}

Agradecemos al Instituto Nacional de Investigaciones Agropecuarias (INIAP), a través del programa nacional de Foresteria, Quito, Ecuador.

\section{References:}

1. Aguilar-Barojas, S. (2005). Fórmulas para el cálculo de la muestra en investigaciones de salud. Salud en Tabasco, 11 (1-2), 333-338.

2. Alonso, J. (2011). Los sistemas silvopastoriles y su contribución al medio ambiente. Revista Cubana de Ciencia Agrícola, 45, (2).

3. Albán, M. A. (2010). Áreas protegidas y deforestación: Una realidad frente al cambio climático. Quito: CEDA, Centro de Derecho ambiental.

4. Anda Basabe, S., Gómez de la Torre, S., \& Bedoya Garland, E. (2017). Estrategias productivas familiares, percepciones y deforestación en un contexto de transición forestal en el caso de Tena en la Amazonía ecuatoriana. Anthropologica XXXV, N. ${ }^{\circ}$ 38, 177-209.

5. Arévalo, V., Andino, M., \& Grijalva, J. (2008). Geopolítica y transformaciones agrarias: El Valle del Quijos en la Amazonia Ecuatoriana. INIAP Publicación miscelánea 142.

6. Cañadas Cruz, L. (1983). El Mapa Bioclimático y Ecológico del Ecuador. Quito: MAG-PRONAREG.

7. Carrasco Carrasco, R. U., Figueredo Calvo, R., Curbelo Rodríguez, L., \& Masaquiza Moposita, D. A. (2017). Caracterización de fincas ganaderas vacunas para el trabajo de extensión rural en Ecuador. I: Determinación de las principales heterogeneidades. Revista de Producción Animal, 29(2),1-5. Recuperado de http://scielo.sld.cu/scielo.php?script=sci_arttext\&pid=S2224$79202017000200001 \& \operatorname{lng}=\mathrm{es} \& \operatorname{lng}=\mathrm{es}$

8. ESPAC. (2017). Encuesta de Superficie y Producción Agropecuaria continua. Quito: INEC.

9. Ferreira, L., \& Tourrand, J. (2002). Caracteriacao e dinámica dos sitemas de producao agrícola más frentes pioneiras ao longo do Arco de Desmatamento da Amazonia. 1 Jornadas Amazonicas. Brasilia: UnB-CDS.

10. Grijalva, J., V. Arévalo, V., \& Barrera, V. (2002). Informe de investigación en el Valle de Quijos y Piedemonte del Ecuador. Proyecto IAI/NSF “Ganadería, uso de la tierra y deforestación en 
zonas amazónicas de Brasil, Perú y Ecuador: estudio comparativo". Universidad de La Florida-INIAP-UNAS-EMBRAPA-Universidad Federal do Pará-McGill University.

11. Grijalva, J., Arévalo, V., \& Wood, C. (2004). Expansión y trayectorias de la ganadería en la Amazonia del Ecuador. Publicación miscelánea del INIAP.

12. Leiva Galarza, T. (2011). Relaciones de género en los sistemas agropecuarios (Tesis de Ingeniería). Quito: Pontificia Universidad Católica del Ecuador.

13. Lyngbaek, A., Muschler, R., \& Sinclair, F. (2001). Productivity and profitability of multistrata organic versus conventional coffee farms in Costa Rica. Agroforestry Systems, 205-213.

14. Mahecha, L. (2003). Importancia de los sistemas silvopastoriles y principales limitantes para su implementación en la ganadería colombiana. Revista Colombiana de Ciencias Pecuarias, 11-18.

15. Mogrovejo Jaramillo, P. (2017). Bosques y cambio climático en Ecuador: El regente forestal como actor clave en la mitigación del cambio climático (Tesis de Maestría). Quito: Universidad Andina Simón Bolívar, Área de Estudios Sociales y Negociación Ambiental.

16. Ochoa, D., \& Valarezo-García, J. (2014). Caracterización y análisis de rentabilidad de los sistemas de producción ganaderos presentes en el cantón Yantzaza, Ecuador. CEDAMAZ, 76-85.

17. Oliveira-miranda, T. (2015). Deforestación en la Amazonía, (Octubre). ed Amazónica de Información Socioambiental Georeferenciada (RAISG).

18. Pérez Espejo, R. (2008). El lado oscuro de la ganadería. Problemas del desarrollo. Prob. Des [online], 217-227.

19. Pérez Sánchez, E. (2006). Caracterización de sistemas silvopastoriles y su contribución socioeconómica a productores ganaderos de Copán. Honduras. Turrialba, Costa Rica: CATIE.

20. Ramachandran Nair, P. (1997). Agroforestería Centro de Agroforestería para el desarrollo sostenible. Revista Chapingo. Serie Ciencias Forestales y del ambiente. México, 101-106.

21. Ríos-Núñez, S., \& Benítez-Jiménez, D. (2015). Análisis del funcionamiento económico productivo de los sistemas de producción cárnica bovina en la Amazonía Ecuatoriana. Arch. Zootec, 409-416.

22. Vargas, J., Benítez, D., Torres, V., Velázquez, F., \& Erazo, O. (2011). Tipificación de las fincas ganaderas en el piedemonte de las provincias Los Ríos y Cotopaxi de la República del Ecuador. Revista Cubana de Ciencia Agrícola, 45, 381-390.

23. Vargas, J., Ríos, S., Torres, V., Benítez, D., Soria, S., Navarrete, H., \& Pardo, D. (2014). Tipificación de las fincas ganaderas de doble 
propósito en la provincia de Pastaza (Characterization of dual-purpose cattle farms in the province Pastaza). Revista Amazónica Ciencia y Tecnología, 183-199.

24. Veiga, J., \& Tourrand, J. (2002). Pecuária na Amazonia: situacao actual e desafios para o futuro. En O futuro da Amazonia: Dilemas, Oportunidades e Desafios no Limiar do Seculo XX. Universidad Federal do Pará.

25. Villavicencio-Enríquez, L. (2013). Caracterización agroforestal en sistemas de café tradicional y rústico en San Miguel, Veracruz, México. Chapingo. Serie Ciencias Forestales y del Ambiente, 19, 6780.

26. Wunder, S. (2000). The economics of deforestation: the example of Ecuador. London, UK: MacMillan and St. Martin Press in association with St. Anthony's College. 